\title{
Radiological Appearances of Lymphomas Arising from Mucosa-associated Lymphoid Tissue (MALT) in the Lung
}

\author{
P. G. O’DONNELL, S. A. JACKSON, K. T. TUNG, B. HASSAN*, B. WILKINS $\dagger$ and G. M. MEAD*
}

Departments of Radiology $\nmid$ Histopathology and ${ }^{*}$ Medical Oncology, Royal South Hants Hospital, Southampton, UK

\begin{abstract}
We review the radiological findings in 13 patients with histologically proven $(n=10)$ or clinically diagnosed $(n=3)$ lymphomas arising in mucosa-associated lymphoid tissue (MALT) of the lung. These rare B-cell lymphomas typically follow an indolent course, and many cases are still being incorrectly described in current radiological literature under the term pseudolymphoma. The patients frequently give a history of autoimmune disease involving the affected organ, and involvement of another mucosal site as part of a disseminated MALT lymphoma, is common. The radiographic patterns of pulmonary parenchymal involvement in lung MALT lymphomas have been reviewed, and correlation made with their clinical behaviour. O'Donnell, P. G., Jackson, S. A., Tung, K. T., Hassan, B., Wilkins, B. \& Mead, G. M. (1998). Clinical Radiology 53, 258-263. Radiological Appearances of Lymphomas Arising from Mucosa-associated Lymphoid Tissue
\end{abstract}

Accepted for Publication 18 July 1997

Non-Hodgkin's lymphomas (NHL) are a complex group of neoplasms which, for the purposes of clinical management and prognosis can be divided into low, intermediate and high grade [1]. Primary lymphomas of mucosa-associated lymphoid tissue (MALT) within the lung are rare, arising from bronchus-associated lymphoid tissue (BALT), which is histologically distinct from true intrapulmonary lymph nodes. They have recently been reclassified as extranodal marginal zone lymphomas [2]. Because the majority of these tumours have an indolent course and relatively benign histological features, many have been described previously using the term pseudolymphoma [3]. This term is now unacceptable because current immunohistochemical and molecular genetic methods show that these tumours contain clonal populations of lymphoid cells, thereby representing true lymphomas. We report 13 patients with histologically proven $(n=10)$ or clinically diagnosed $(n=3)$ lung MALT lymphomas, with a review of their radiological appearances and evolution.

\section{PATIENTS AND METHODS}

We reviewed the radiographic findings of patients with histologically proven or clinically diagnosed lung MALT lymphomas presenting to our centre over the past 14 years. Thirteen cases of MALT lymphoma of the lung were identified, with an age range 44-75 years (mean, 66 years), and an uneven distribution of sex (11 females to two males).

\section{RESULTS}

\section{Presentation and Histology}

The clinical presentation of eight of the patients was with cough; the remaining five patients initially had no chest

\footnotetext{
Correspondence to: Dr K. T. Tung, Department of Radiology, Brintons Terrace, Graham Road, Royal South Hants Hospital, Southampton SO9 4PE, UK.
}

symptoms (Table 1). Histology was obtained in three patients using thoracotomy and lung resection as an initial diagnostic procedure (segmentectomy in one patient and lobectomy in two), with bronchoscopic or image-guided biopsy in a further seven patients. The remaining three patients had histological evidence of MALT lymphoma at another mucosal site and appropriate chest imaging findings consistent with the diagnosis of disseminated MALT lymphoma. In the latter cases, there was improvement or resolution of the pulmonary abnormalities coincident with a clinical response to treatment. The primary site involved in these three cases was the stomach, salivary gland and thyroid.

A total of seven of the 13 patients studied had a history of mucosal involvement at another site at some stage of the disease. This pre-dated the diagnosis of lung MALT lymphoma in five and was seen at extrathoracic mucosal sites following the diagnosis of the lung abnormality in the remaining two. The time course of these disseminated MALT lymphomas is summarized in Table 1.

Five of the 13 patients had a history of autoimmune disease or positive tests for autoantibodies (see Table 1).

Of the 10 lung MALT lymphomas for which histology was available, eight were low grade and two intermediate grade at diagnosis [1]. In the three patients in whom lung MALT lymphoma was clinically diagnosed, biopsy of the primary site affected showed the parotid MALT lymphoma to be mixed intermediate and low grade, and the thyroid and stomach tumours to be low grade. A total of 10 of our patients were therefore low grade at diagnosis; transformation to intermediate grade disease was seen subsequently in four.

\section{Radiographic Appearances \\ Chest Radiograph}

Initial chest $\mathrm{X}$-ray appearances varied; six patients presented with peripheral consolidation (Fig. 1), four patients demonstrated dense perihilar consolidation (Fig. 2), and three cases presented with multiple pulmonary masses, 


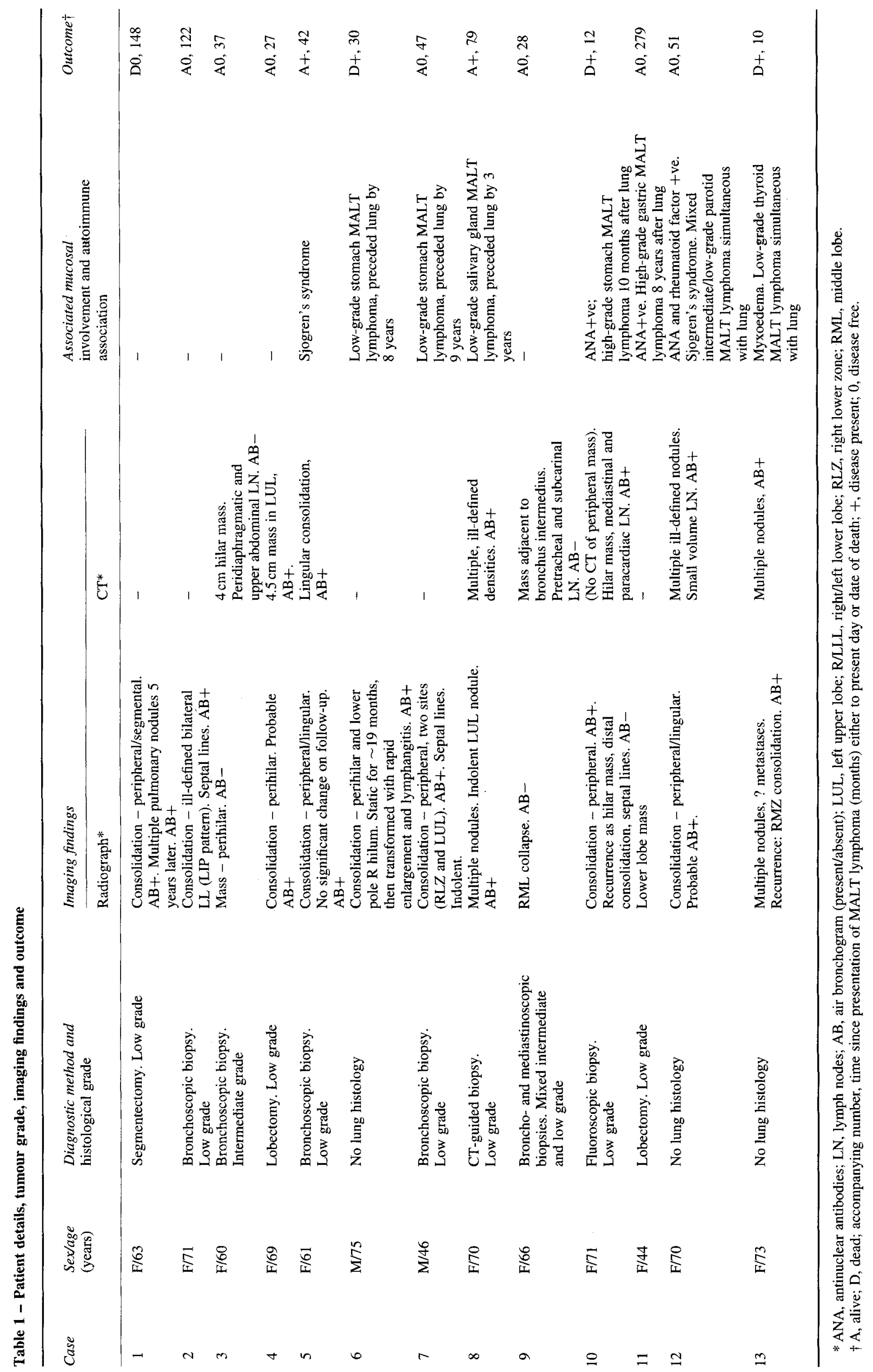




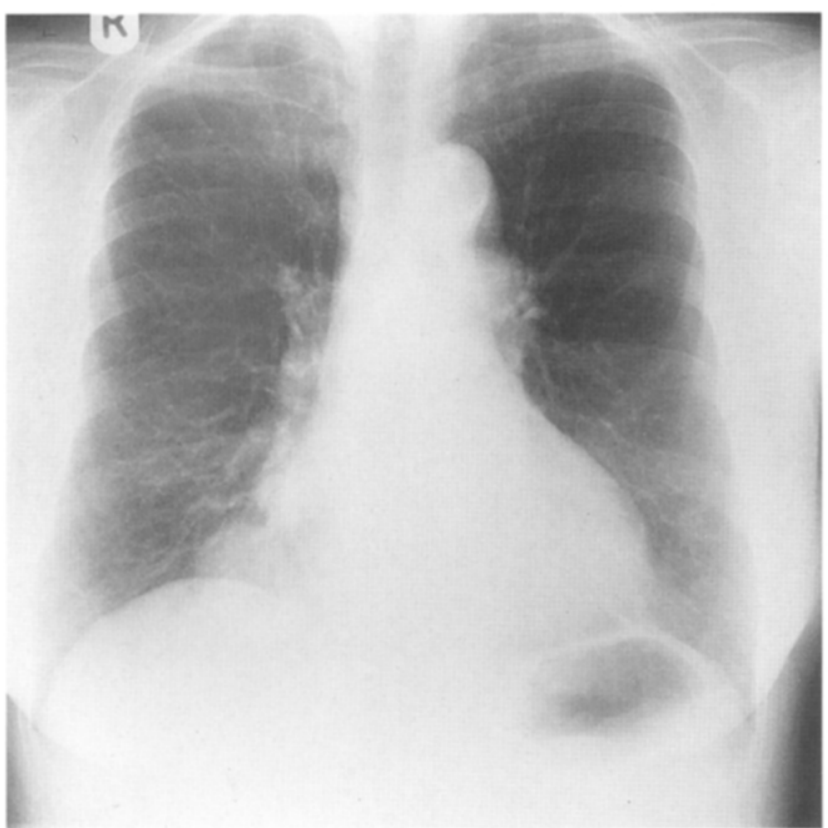

Fig. 1 - Case 10. Frontal radiograph showing peripheral pattern of MALT lymphoma, with well-defined lower zone consolidation and air bronchogram.

which were generally rather ill-defined. This includes the representation of case 1 , who initially presented with basal consolidation. A prominent feature of all lesions was the presence of an air bronchogram. This was seen on the plain radiograph in five out of the six patients with peripheral consolidation. Air bronchograms appeared less prominent in the central/perihilar consolidations, being seen in two out of four cases. An air bronchogram was not suspected in the remaining two patients in this group, and its absence was confirmed by CT. Air bronchograms were also demonstrated on chest radiograph in all patients with multiple

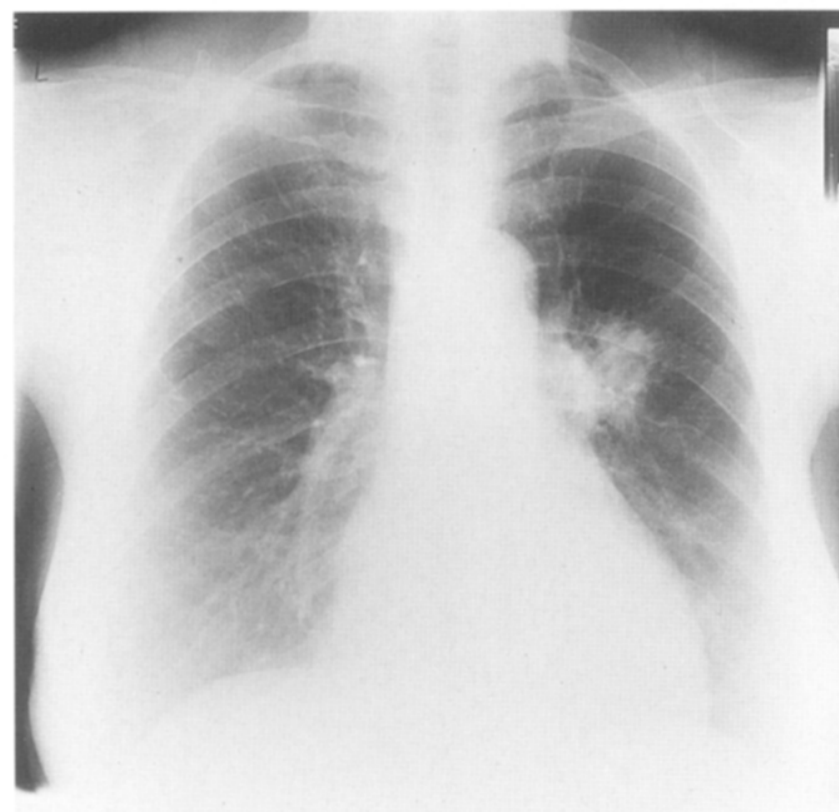

Fig. 2 - Case 4. Frontal radiograph showing left perihilar consolidation and probable air bronchogram. Lobectomy specimen confirmed MALT lymphoma (see Fig. 6).

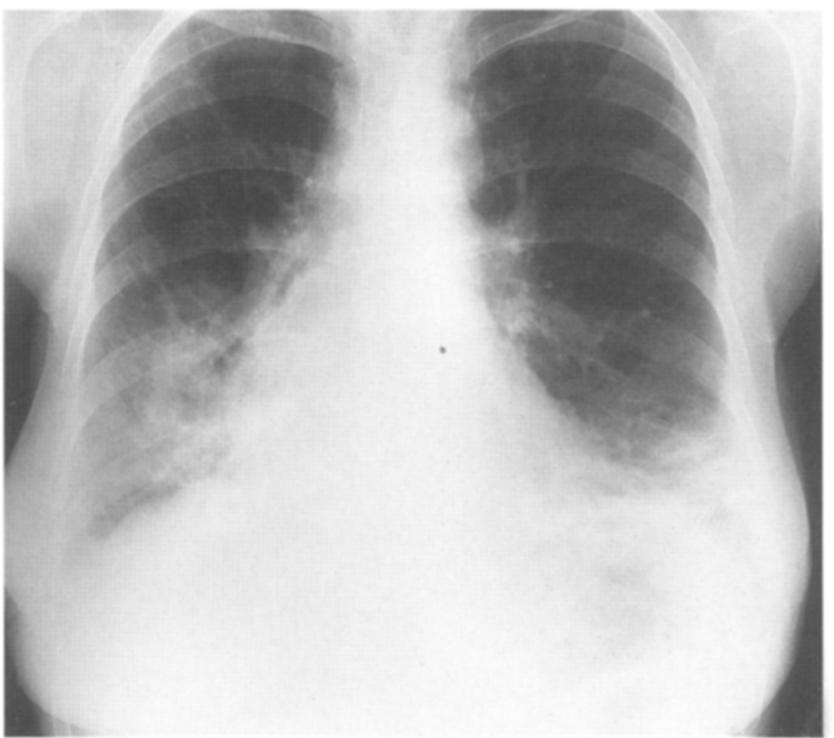

Fig. 3 - Case 2. Frontal radiograph showing diffuse lower zone consolidation (LIP pattern), which in our series, was an uncommon radiographic appearance (biopsy-proven pulmonary MALT lymphoma).

pulmonary masses, including the second presentation of case 1. One patient demonstrated more diffuse lower lobe consolidation (lymphocytic interstitial pneumonitis or LIP pattern, Fig. 3), and in this case, patchy lucency within the pulmonary shadowing suggestive of an air broncho- or bronchiologram was present. In addition, septal lines were prominent in the areas of abnormality, consistent with a mixed interstitial and air-space pattern of disease. Septal lines were also demonstrated in association with both a perihilar and peripheral (case 7, Fig. 4) MALT lymphoma. Linear densities suggesting lymphangitis, radiating from a perihilar tumour mass, was seen in one case, which was associated with a rapidly enlarging, intermediate grade MALT lymphoma and disseminated disease (Fig. 5).

One patient presented with middle lobe collapse secondary to perihilar tumour.

The areas of consolidation persisted for a prolonged

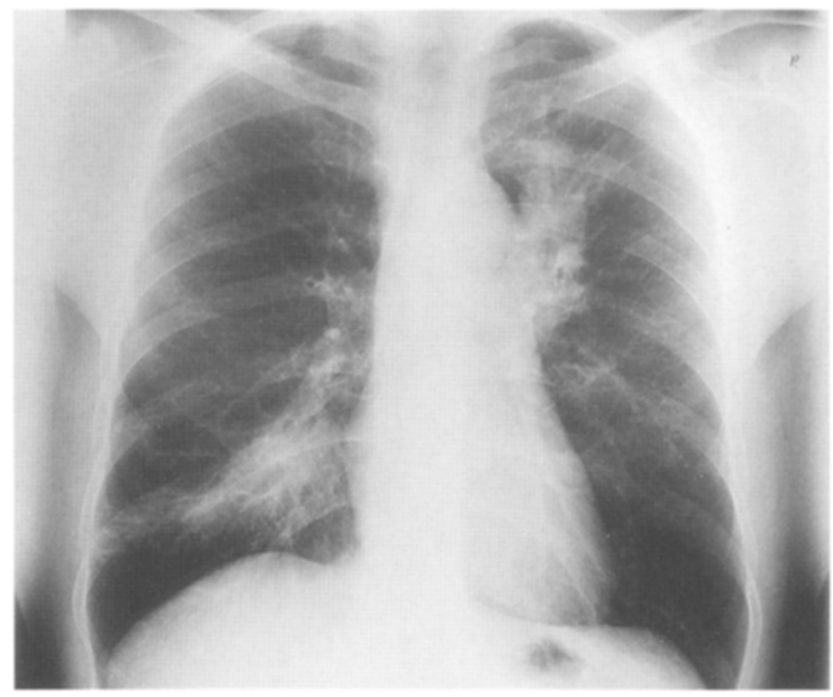

Fig. 4 - Case 7. Frontal radiograph showing consolidation at two sites: left upper lobe and right lower zone. Prominent septal lines associated with the lower zone tumour. This tumour, particularly the upper lobe moiety, also displayed relative indolence over 48 months. 


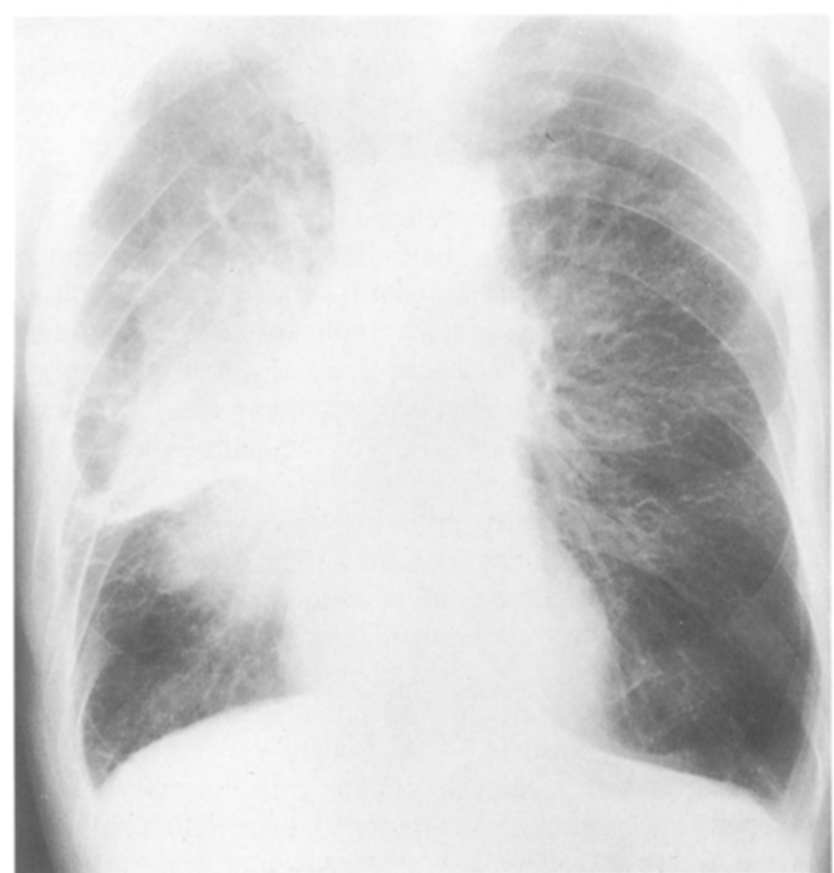

Fig. 5 - Case 6. Frontal radiograph showing a large right perihilar mass with radiating linear densities suggesting lymphangitis. The tumour was, at the time of examination, rapidly enlarging, having been static in appearance for 19 months.

period (range 19-72 months, mean 46.4 months) in a number of cases $(n=5)$, with either no change or slow growth, suggesting indolence. All patterns of pulmonary involvement showed indolent behaviour.

\section{Computed Tomography}

Standard axial computed tomography (CT) was performed in eight of the 13 patients. CT confirmed the presence of air bronchograms in all cases where it was suspected $(n=2)$, or definitely seen ( $n=3$, Figs $6 \& 7$ ) on radiographs, and confirmed the absence of bronchograms in two patients, both of whom had perihilar tumours. In one patient, who had

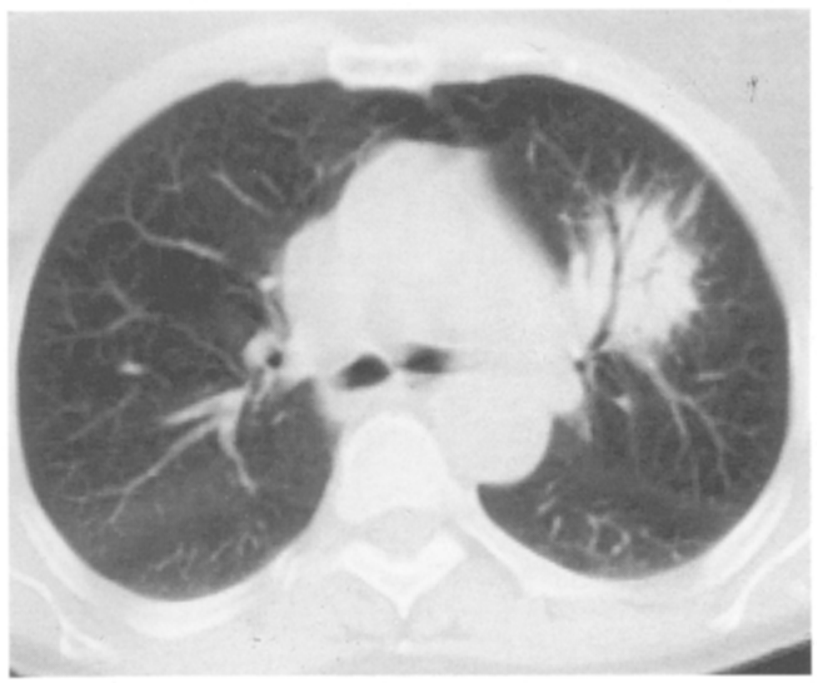

Fig. 6 - Case 4. Axial CT section (lung windows) demonstrating typical dense consolidation in a perihilar MALT lymphoma, in this case, left upper lobe, with prominent air bronchogram. (See Fig. 2 for corresponding radiograph.)

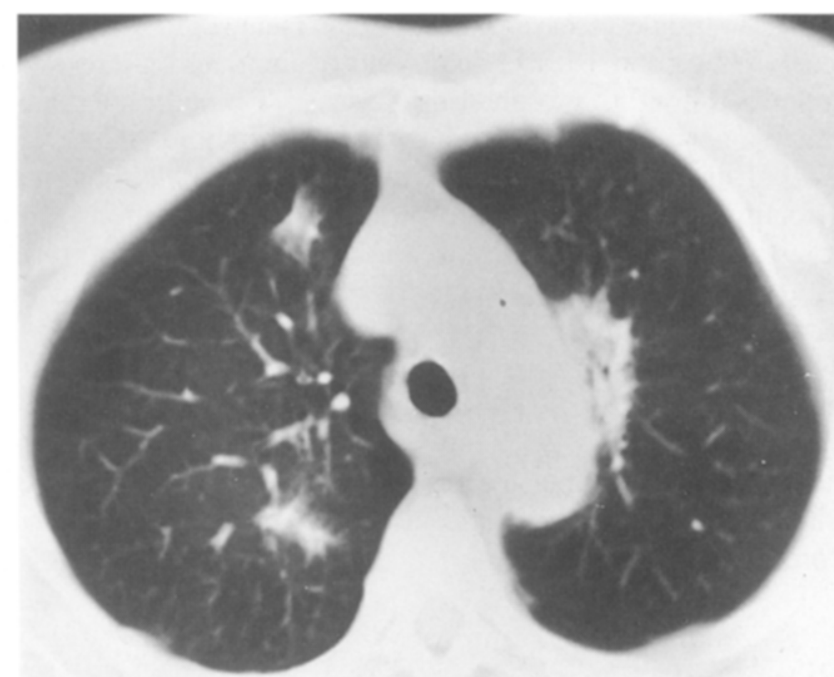

Fig. 7 - Case 8. Axial CT section (lung windows) demonstrating air bronchograms within multiple MALT lymphoma nodules.

a perihilar tumour, bronchograms were visible only on CT (case 10 recurrence). In another, CT was responsible for correctly diagnosing the pattern of pulmonary involvement as multiple nodules with air bronchograms, in a patient thought to have lingular consolidation on chest radiograph. In all cases, CT defined the extent and distribution of the disease with greater clarity. For example, in case 8 , CT identified a larger lower lobe nodule not visible on radiograph, which was amenable to CT-guided biopsy. In case 9, CT suggested the presence of a central mass in a patient whose radiograph revealed only middle lobe collapse. MALT lymphoma was confirmed on bronchoscopy, with tumour infiltrating the anterior wall of bronchus intermedius. CT was particularly useful for further assessment of central and multiple masses, and allowed clearer demonstration of air bronchograms (seen in six of the eight patients who had chest CT).

Mediastinal lymphadenopathy was not suspected from plain radiographic appearances in any case. Small volume mediastinal lymphadenopathy was only seen in patients with more aggressive disease, occurring in $66 \%(n=4)$ of our patients with intermediate grade MALT lymphoma, with no large nodal masses seen, in keeping with the predominantly extranodal nature of this lymphoma. In no patients was definite cavitation seen.

\section{Clinical Management and Outcome}

The management varied: three patients were treated by resection of a lobe $(n=2)$ or segment $(n=1)$, which was performed as part of the initial management of an undiagnosed pulmonary mass. Seven patients were treated with chemotherapy, either oral $(n=5)$, or intravenous $(n=2)$. Two patients were treated with a combination of chemotherapy and radiotherapy. Case 1 , having initially undergone basal segmentectomy, received chemotherapy when she re-presented with multiple pulmonary nodules. One patient (case 5), who presented in 1993, is being managed conservatively, and has received no treatment to date.

Nine of our 13 patients are alive at the time of writing; four are dead. Seven patients are alive with no evidence of disease a range of 27 months to 14 years following presentation (mean 84 months), and two patients remain alive 
with low-grade disease which is being managed conservatively. Four patients are dead, one of unrelated causes, 70 months following presentation (case 1). Two other patients died as a direct result of their MALT lymphoma. Case 10 died from aggressive disseminated MALT lymphoma affecting stomach and lung, following transformation from low-grade pulmonary disease. Case 13 , whose low-grade thyroid and lung MALT lymphoma had initially responded, died following transformation with recurrence in both lungs. The other death in our group, case 6 , who had responded to treatment for gastric MALT lymphoma some years previously, relapsed with a lung mass, which, at the time of death, was enlarging rapidly. Unfortunately, this patient died at another hospital and his cause of death can not be confirmed.

\section{DISCUSSION}

Pulmonary parenchymal involvement in lymphoma is rare. It is more common in Hodgkin's disease than NHL. A previously published series of 300 untreated cases of lymphoma [4] described lung involvement in $11.6 \%$ of patients with Hodgkin's disease, compared with only $3.7 \%$ of patients with NHL.

The nomenclature of lymphoid proliferations of the lung has been rather confused, including lymphocytic lymphoma, LIP and pseudolymphoma. The entity 'primary pulmonary lymphoma', described in 1963 in Saltzstein in 102 cases, had a relatively good prognosis in comparison to other pulmonary tumours and other forms of malignant lymphoma. Saltzstein interpreted the low incidence of dissemination in these cases as evidence that some of the pulmonary lesions were not true lymphomas but examples of a benign chronic inflammatory process involving the pulmonary parenchyma, which he termed pseudolymphoma [3]. He did, however, warn that histological differentiation between a malignant lymphoma and pseudolymphoma was not always possible. Since then, the introduction of immunohistochemical and molecular genetic methods has confirmed that most of these tumours are, in fact, low-grade monoclonal B-cell lymphomas [5-8].

The distinct clinico-pathological entity of low-grade Bcell lymphomas arising from mucosa-associated lymphoid tissue (MALT) was first described in 1983 in a patient with primary gastrointestinal lymphoma [9]. Since then further reports have been published of similar tumours, of both low and intermediate grade, which have arisen from other organs including the lungs, salivary glands, thyroid gland, thymus, and less commonly, the orbit, bladder and genitourinary tract $[10-12]$. Within the lung, these tumours can be demonstrated to arise from bronchus-associated lymphoid tissue (BALT), which is distinct from the lymphoid tissue within lymph nodes. In addition, MALT-derived tumours tend to remain extranodal, although lymph nodes can be involved in advanced disease. They also tend to remain localized to the tissue of origin but, when spread occurs, involve other organs where extranodal lymphoid tissue is found. This pattern of spread can be explained in terms of a distinct population of lymphocytes 'belonging' to mucosal sites around the body, with specific circulation and homing properties [13]. Many patients who develop MALT lymphoma have a history of inflammatory or autoimmune disease in their organ of origin, for example, Helicobacter gastritis, Sjogren's syndrome and Hashimoto's thyroiditis [2]. Histologically, MALT lymphomas demonstrate a characteristic appearance with reactive B-cell follicles surrounded or infiltrated by small centrocyte-like tumour cells together with the presence of lympho-epithelial lesions (Fig. 8). There is frequently evidence of plasma cell differentiation.

The radiological manifestations of pulmonary parenchymal disease in lymphoma have been classified previously into four patterns: nodular (with or without cavitation), pneumonic, bronchovascular/lymphangitic, and miliary $[14,15]$. However, these descriptions are of limited utility as overlap is commonly seen between these various patterns of disease [16]. In our experience, the radiological manifestations of pulmonary MALT lymphomas are often highly suggestive. They usually consist of areas of dense focal consolidation, which are often single, with peripheral lesions (Fig. 1) occurring more commonly than perihilar ones. Review of the literature with reference to the appearances of these tumours suggests that they present commonly as single or multiple nodules in either a perihilar or more peripheral distribution [17-19]. Poorly defined pulmonary consolidation can occur [20]. If the consolidation appears diffuse, a feature which has previously been called LIP (Fig. 3 ), it is likely to reflect parenchymal extension of MALT lymphoma from peribronchial/peribronchiolar regions [6]. There appears to be no lobar predilection and air bronchograms are a well recognized feature, particularly on CT, irrespective of pattern or distribution of tumour. In our series, areas of consolidation persisted over a prolonged period in a number of cases, suggesting an indolent course. Slowly progressive pulmonary consolidation in conjunction with a supportive history of autoimmune disease or lymphoma in another mucosal site should strongly suggest the diagnosis, as the radiographic features are not diagnostic in isolation. Indeed, the imaging findings in one of our cases, case 4, should have suggested that non-surgical management was the most appropriate, but histology was finally obtained from the resected left upper lobe. More aggressive tumours, and transformation from low to intermediate grade are, however, possible. In these cases, mediastinal lymphadenopathy is often a feature.

The imaging features described in our series of pulmonary MALT lymphomas, particularly those of low-grade tumours, are identical to previous radiological descriptions

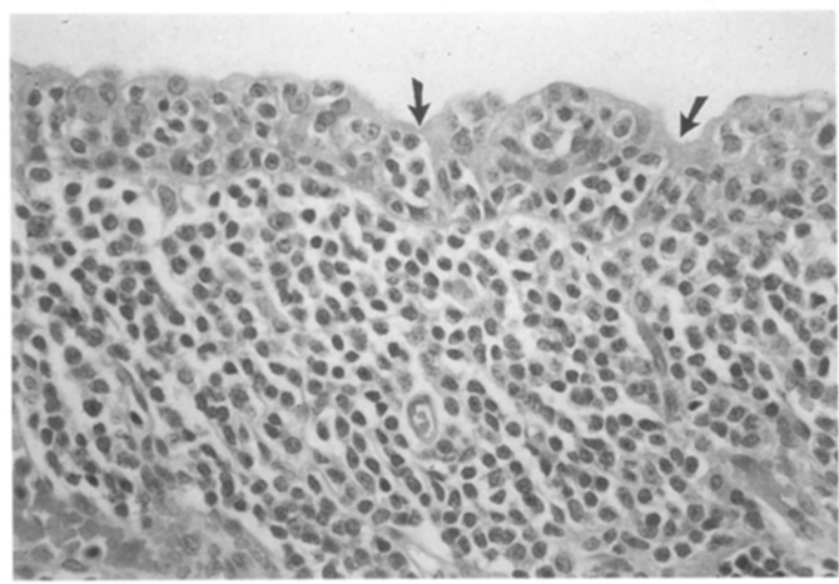

Fig. 8 - Low-grade MALT lymphoma of the lung showing numerous clusters of small lymphoid cells within bronchial epithelium (lymphoepithelial lesions), highlighted by arrows, and diffuse submucosal infiltration by similar, centrocyte-like cells. Note that a proportion of the lymphoid cells have well-defined pink/lilac cytoplasm indicating plasmacytic differentiation (haematoxylin and eosin stain, original magnification $\times 40$ ). 
of pseudolymphoma. We agree with previous reports that the latter term is inaccurate, and that most tumours previously referred to as pseudolymphomas would be diagnosed as MALT lymphomas using modern laboratory techniques. Previously used laboratory methods have been unable to diagnose the clonality of these tumours accurately. They have relied on demonstration by immunohistochemistry of expression of a single immunoglobulin light chain type by tumour cells. However, this cannot always be achieved using fixed tissues embedded in paraffin. Such tissue is often the only material available in retrospective studies [8]. The presence of reactive follicles and plasma cells within MALT lymphoma masses, in addition to the monoclonal malignant population, may have caused their overdiagnosis as benign lymphoid proliferations on the basis of apparent polyclonality [6]. Immunohistochemical methods for the detection of surface immunoglobulin using frozen tissue or fresh cell suspensions are more sensitive, allowing tumours to be diagnosed more accurately if such material is available [6]. Some groups have reported gene rearrangement studies as useful in the distinction of pseudolymphoma from lymphoma [21,22]. However, no reports of gene rearrangement in pseudolymphoma have been found in our search of the literature.

In summary, pulmonary MALT lymphomas comprise a distinct group of extranodal lymphomas. They usually have characteristic histological features and an indolent clinical course. Low-grade tumours appear to correspond to lesions described previously as pseudolymphomas, although modern techniques have shown most, if not all, of these tumours to represent true low grade B-cell lymphomas. In our series, radiographic findings were often highly suggestive, with areas of dense, persistent consolidation. Air bronchograms were present in the vast majority. The presence of mediastinal lymphadenopathy may suggest more aggressive disease. The diagnosis of MALT lymphoma should be considered in patients who have dense, persistent consolidation unresponsive to usual measures. A history of previous autoimmune disease or even MALT lymphoma at another site, may be present and provides a further clue to diagnosis. CT may be required to demonstrate the air bronchograms, give further information on the distribution and morphology of the lesions, and provides guidance for diagnostic percutaneous biopsy.

Acknowledgments. Many thanks to Dr A. Herbert, of the Department of Histopathology, Southampton General Hospital, for advice given in the preparation of this paper.

\section{REFERENCES}

1 National Cancer Institute sponsored study of classifications of nonHodgkin's lymphomas: Summary and description of a working formulation for clinical usage. Cancer 1982;49:2112-2135.

2 Harris NL, Jaffe ES, Stein $\mathrm{H}$ et al. A revised European-American classification of lymphoid neoplasms: a proposal from the International Lymphoma Study group. Blood 1994;84:1361-1392.

3 Saltzstein SL. Pulmonary malignant lymphomas and pseudo-lymphomas: classification, therapy and prognosis. Cancer 1963;16:928-955.

4 Filly R, Blank N, Castellino RA. Radiographic distribution of intrathoracic disease in previously untreated patients with Hodgkin's disease and non-Hodgkin's lymphoma. Radiology 1976;120:277-281.

5 Li G, Hansmann M, Zwingers T, Lennert K. Primary lymphomas of the lung: morphological immunohistochemical and clinical features. Histopathology 1990;16:519-531.

6 Addis BJ, Hyjek E, Isaacson PG. Primary pulmonary lymphoma: a reappraisal of its histogenesis and its relationship to pseudolymphoma and lymphoid interstitial pneumonia. Histopathology 1988;13:1-17.

7 Julsrud PR, Brown LR, Chin-Yang L et al. Pulmonary processes of mature-appearing lymphocytes: pseudolymphoma, well-differentiated lymphocytic lymphoma, and lymphocytic interstitial pneumonitis. Radiology 1978;127:289-296.

8 Herbert A, Wright DH, Isaacson PG et al. Primary malignant lymphoma of the lung: histopathologic and immunologic evaluation in nine cases. Human Pathology 1984;15:415-422.

9 Isaacson PG, Wright DH. Malignant lymphoma of mucosa-associated lymphoid tissue. Cancer 1983;52:1410-1416.

10 Isaacson $P G$, Wright $D H$. Extranodal malignant lymphoma arising from mucosa associated lymphoid tissue. Cancer 1984;53:2515-2524.

11 Pelsting RJ, Essell JH, Kurtin PJ et al. Diversity of organ site involvement among malignant lymphomas of mucosa-associated tissues. American Journal of Clinical Pathology 1991;96:738-745.

12 Isaacson PG. Lymphomas of mucosa-associated lymphoid tissue (MALT). Histopathology 1990;16:617-619.

13 Pals ST, Horst E, Scheper RJ, Meijer CJLM. Mechanisms of human lymphocyte migration and their role in the pathogenesis of disease. Immunology Reviews 1989;108:111-133.

14 Balikan JP, Herman PG. Non-Hodgkin lymphoma of the lung. Radiology 1979;132:569-576.

15 Jackson SA, Tung KT, Mead GM. Multiple cavitating pulmonary lesions in non-Hodgkin's lymphoma. Clinical Radiology 1994;49:883-885.

16 Lewis ER, Caskey CI, Fishman EK. Lymphoma of the lung: CT findings in 31 patients. American Joumal of Roentgenology 1991;156:711-714.

17 Wotherspoon AC, Soosay GN, Diss TC et al. Low grade primary B-cell lymphoma of the lung. American Journal of Clinical Pathology 1990; 94:655-660.

18 Roggeri A, Aostini L, Vezzani G. Primary malignant non-Hodgkin's lymphoma of the lung arising in mucosa-associated lymphoid tissue (MALT). European Respiratory Journal 1993;6:138-140.

19 Canver CC. Primary B-cell malignant lymphoma of the lung. Annals of Thoracic Surgery 1993;56:964-966.

20 Liaw YS, Yang PC, Su IJ. Mucosa-associated lymphoid tissue lymphoma of the lung with cold-reacting autoantibody mediated haemolytic anaemia. Chest 1994;105:288-290.

21 Shihota T, Chiba W, Ikeda S, Ikei N. Gene analysis in pulmonary pseudolymphoma. Chest 1993;103:335-338.

22 Aoki $T$, Watanabe M, Takagi K, Tanaka S, Aida S. Gene rearrangement studies on lymphoma of the lung: report of a case. Surgery Today (Japan) 1995;25:281-283. 